\title{
THE EFFECT OF ROTOR BALANCE DURING CRUSHING OF INTERMETALLIC COMPOUNDS IN HAMMER CRUSHERS
}

\author{
${ }^{1}$ Yurii KOBRIN, ${ }^{2}$ Andrii VLASOV, ${ }^{3}$ rene SHEVCHENKO \\ ${ }^{1}$ National Metallurgical Academy of Ukraine, Dnipro, Ukraine, ku 76@yahoo.com \\ 2Zaporizhzhia National University, Zaporizhzhia, Ukraine, vlasov-aa@znu.edu.ua \\ 3Zaporizhzhia National University, Zaporizhzhia, Ukraine, iashevia@yahoo.com
}

https://doi.org/10.37904/metal.2020.3617

\begin{abstract}
The paper considers and presents such factors and parameters affecting the crushing of intermetalides in hammer crushers as - imbalance and balance of the rotor. The structural and technological factors of impact crushing equipment are analyzed. These factors affect not only the energy performance of grinding, but can also lead to increased wear of parts and components of the hammer crusher. Methods of improvement by prompt and timely balancing of the rotor in a hammer mill and on a balancing stand are proposed. Research objective: crushing of intermetalides and modeling of the operation of hammer crushers, namely: the destruction of crushed materials and operating conditions with and without vibration due to an unbalanced rotor and a balanced rotor in order to understand how much this affects the performance of hammer crushers and energy loss. It is also necessary for the initial assessment and selection of design and technological parameters for: design, operation and repair of impact crushing equipment - in order to optimize the cost of electric energy for the design of new hammer crushers and those in operation, as well as to increase the time without repairs all crusher parts. With balancing, it was possible to balance the rotor. After conducting research at the laboratory facility, the percentage ratio of the negative influence of the construction factor was determined, which is from $8 \%$ to $15 \%$ of the energy overrun, namely, the rotor imbalance.
\end{abstract}

Keywords: Crusher, intermetallic, rotor, unbalanced, balancing

\section{INTRODUCTION}

The amount of energy consumed in the world for crushing in material crushers is approximately $5 \%$. Balancing the balance of power and, accordingly, the balanced use of the necessary resources will lead to a reduction in costs. Hammer crushers belong to impact crushers in which the crushed material is destroyed under the influence of the shock load acting on it by an electric drive through hammers, which are movable and rotate quickly. The crushed material is destroyed from the impact, and from friction - with the help of the same hammers and sieves, which leads to excess costs [1].

Known factors that affect the work - it is technological, technical and structural [1,2].

Technological - these are the properties of crushed material, such as strength, moisture, grain size, brittleness. Technical - frequency of service and its quality.

Structural - this is what materials are used in the manufacture of working elements crushed and other critical elements, what shape these work elements and the dimensions of the workspace.

For a better understanding, consider the design of the hammer crusher, which is shown in the Figure 1, where it is shown: 1 - body, 2 -armor, 3 - motor shaft, 4 -rotor, 5 - hammer, 6 - sieve, 7 - coupling, 8 - motor, 9 - loading hole. 


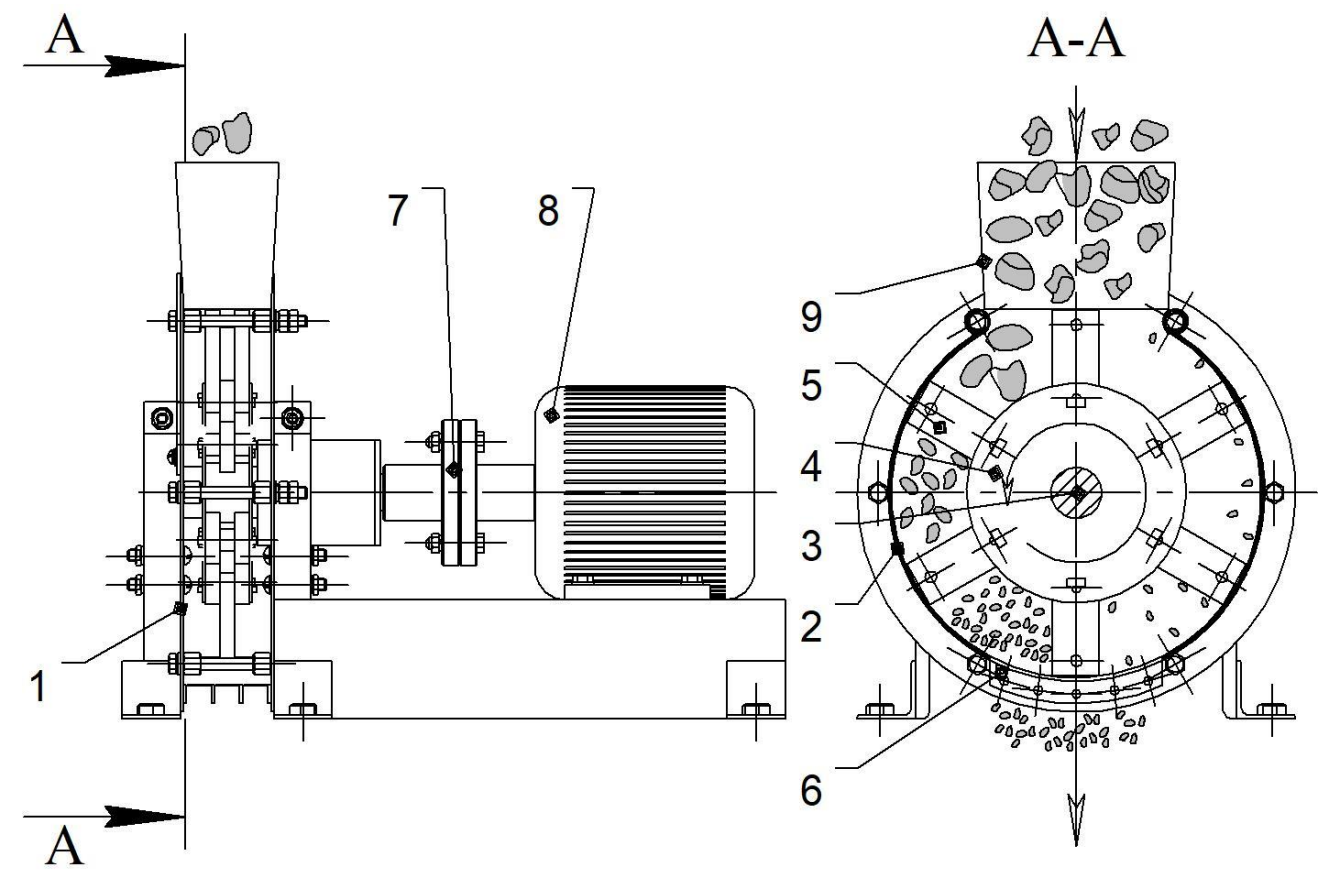

Figure 1 Hammer crusher design

The working elements involved in the direct grinding process are hammers, a sieve and armor. Their shape, weight, size have a direct impact on performance and power consumption.

The technological factor affecting productivity and power consumption, which develops into a structural one, is the occurrence of rotor vibrations caused either by the initial structural disadvantage of the rotor during the manufacture of its components or during assembly, or by the wear of hammers.

Since hammers operate under shock loads and abrasive wear, and the supply of crushed material along the length is uneven, due to the heterogeneity in size and strength characteristics, uneven wear of the hammers along the length of the rotor occurs. Extreme hammers wear out more intensively and this leads to an imbalance of the rotor. Since the hammers are masses rotating around the axis of rotation of the shaft, they under the action of centrifugal force cause a displacement of the center of mass of the rotor, which, in turn, begins to hit the crusher bearings in the radial direction of rotation with an alternating load.

In addition to the negative impact of radial impact on the strength characteristics of the mechanical elements of the crusher and drive, there is also an increased energy consumption. These data were obtained during experiments in a laboratory setup [3].

To solve such a negative factor as the displacement of the center of mass relative to the axis of rotation, and as a consequence of the shock force and increased energy consumption, it is necessary to use the balancing of an unbalanced rotor. Shaft requirements are given in ISO 21940-11: 2016 [4].

The influence of the imbalance of the parts that rotate on different mechanisms, the analysis of these actions and solutions to this problem are considered in scientific works of different areas of science and allow us to take this experience into account [5-8].

\section{RESEARCH REVIEW}

There are three types of balancing of unbalanced rotating masses, these are static, dynamic and general [9].

Static balancing of a pre-assembled, unbalanced rotor can be carried out both on a special stand and in the crusher housing with bearings. Before carrying out static balancing, it is necessary to fix all the hammers to 
their working position, that is, to the position where the centrifugal force deflects them as far as possible from the shaft of the crusher rotor. Fastening is carried out using wooden wedges or other similar elements that are identical in shape and size, which can be removed after static balancing. When fixing hammers with wooden wedges, it is necessary to arrange the wedges symmetrically to the axis of the rotor shaft so as not to introduce even greater imbalance in the rotor of the drawer. After the hammers are fixed, the rotor 4 of the crusher 1 (Figure 1) is conditionally divided into two halves 3 , the half is conventionally called: $\mathrm{H}-$ the heavy part, $\mathrm{L}-$ the light part (Figure 2a). By turning the crusher rotor 90 degrees in any direction, at the maximum possible distance from the axis of the shaft at point $A$, a load $G_{w}$ of such a weight is fixed that the rotor shaft of the crusher, when turning, returns to its original position by an angle of only 10-15 degrees (Figure $\mathbf{2 b}$ ).

After selecting the $\Delta G_{w}$ load with the weight necessary so that the crusher rotor becomes an unbalanced part in the opposite direction, we determine the weight of the compensation weight necessary to eliminate this

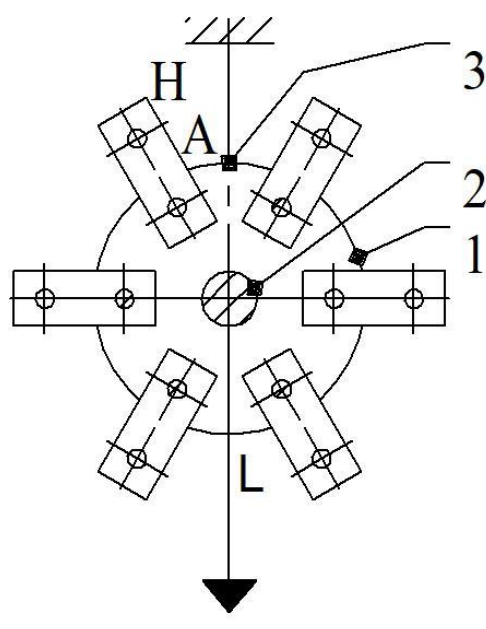

a

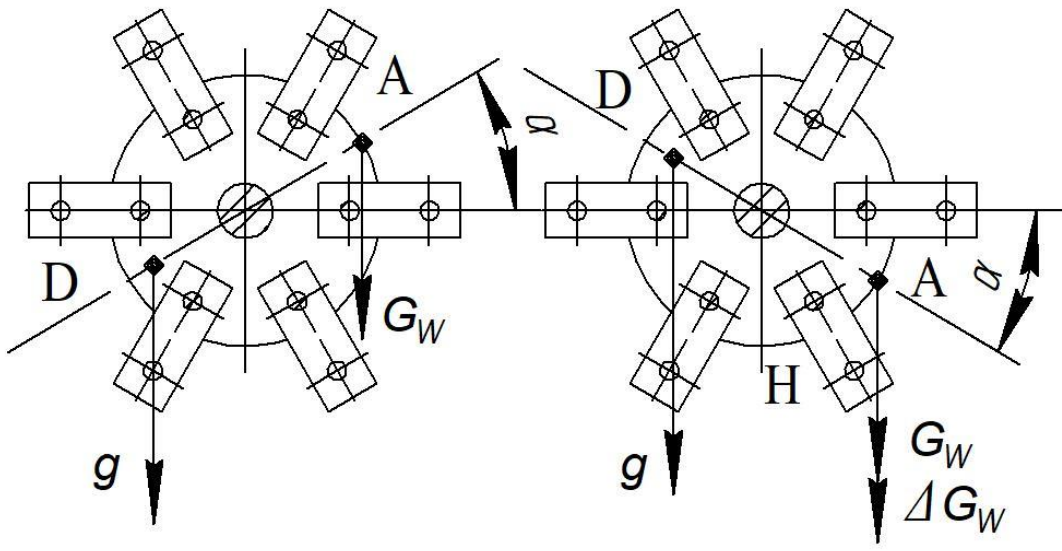

b
C

Figure 2 Static balancing scheme for the crusher rotor

Static balancing, despite its ease of implementation, is not suitable for a hammer mill rotor. The reason for this is the movably suspended hammers. A lot of time is spent on securing them, and additional fasteners for fixing these hammers introduce errors in measurements and calculations, which was shown during the experiment.

More accurate and convenient - this is dynamic balancing bypass load method. Its advantage is - with the help of a trial load. Balancing can be carried out directly in the crusher, having previously installed the crusher on elastic supports with measuring equipment. If the supports and equipment are installed on an ongoing basis - this allows you to fine-tune the hammer crusher without removing the rotor to transfer it to the balancing machine and vice versa. This allows you to reduce the time for maintenance, and accordingly to reduce the technical factor that negatively affects the performance of the crusher.

The essence of dynamic balancing by way of bypassing with a test load and its adaptation to use in hammer crushers is: we take for the balancing surfaces the extreme lateral surfaces of the rotor, individually, on which the hammers are attached and cause slight rotor vibrations with the help of the test load (Figure 3).

The weight of the test load, equation (6) is determined by the formula of its centrifugal force of equation (1)

$$
\begin{aligned}
& P_{T L}=k \cdot G \cdot G_{T L} \cdot r_{1} \cdot \omega^{2} / g \\
& G_{T L}=k \cdot\left(G /\left(r_{1} \cdot \omega^{2}\right)\right)
\end{aligned}
$$


where:

$P_{T L}$ - is the centrifugal force caused by the test load;

$k=0.02-0.03$ is the dynamic coefficient;

$g-$ is the gravity acceleration $\left(\mathrm{m} / \mathrm{s}^{2}\right)$;

$r_{1}-$ is the distance from the rotor axis of rotation to the test load installation location $(\mathrm{mm})$;

$\omega-$ is angular velocity $\left(\mathrm{s}^{-1}\right)$;

$G_{T L}-$ test load weight $(\mathrm{N})$.

After calculating the weight of the test load, it is installed on one side of the extreme lateral surface of the rotor, the angular speed working for the crusher rotor is set, and the readings from instruments located in elastic supports on the side of the test load are recorded. At the same time, supports located on the side opposite to the place of the test load to be installed must be rigidly fixed. These steps must be repeated at all points (Figure 3).

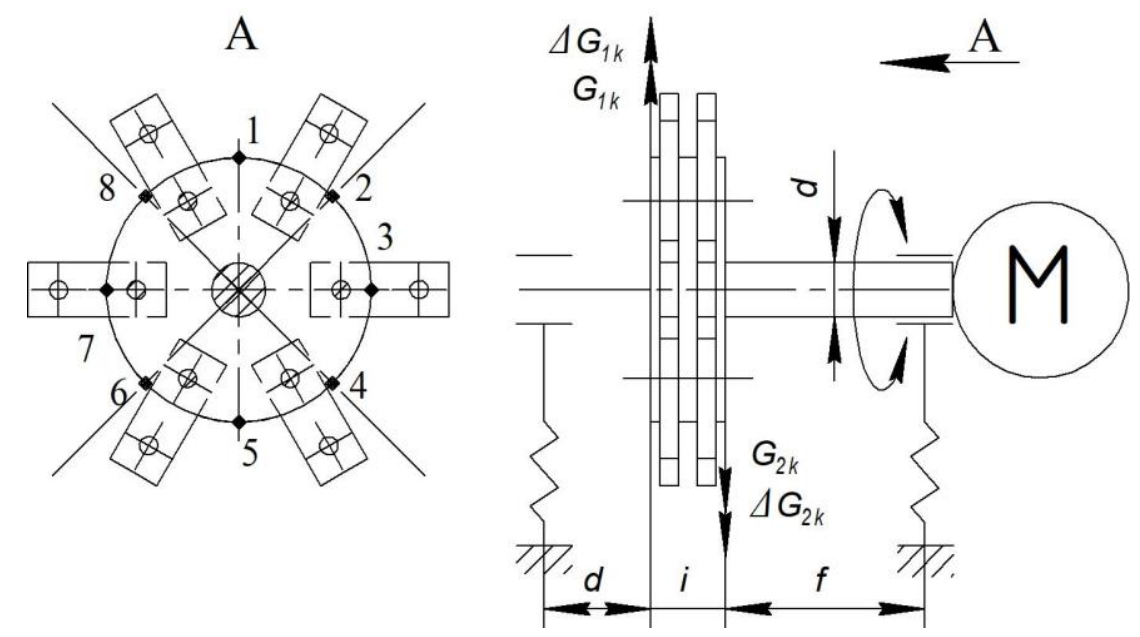

Figure 3 Dynamic balancing scheme for the crusher rotor

According to the data obtained, a graph is constructed according to eight parameters of the amplitude of oscillations on one side of the rotor. When analyzing the graph, it can be seen that the rotor is unbalanced and the maximum and minimum values are visible on the curves on both sides (Figure 4).

At a point with a minimum amplitude parameter, a balancing load calculated by the equation (3)

$$
G_{1 k}=G_{T L} \cdot\left(\left(A_{1 \max }+A_{1 \min }\right) /\left(A_{1 \max }-A_{1 \min }\right)\right)
$$

where: $A_{1 \max }, A_{1 \min }$ - are the maximum and minimum oscillation amplitudes after installing a test load for measurement on one side $(\mathrm{mm})$ (Figure 4)

$G_{T L}-$ is the weight of the test load (N)

The next step is to repeat the previous steps with the test load, only on the opposite side of the crusher rotor.

According to the data obtained, a graph is constructed according to eight parameters of the amplitude of the oscillations on the second side of the rotor and analytically identifies the maximum and minimum values of the amplitude parameters. A balancing weight calculated by the formula is set to a point with a minimum amplitude parameter

$$
G_{2 k}=G_{T L} \cdot\left(\left(A_{2 \max }+A_{2 \min }\right) /\left(A_{2 \max }-A_{2 \min }\right)\right)
$$

where: $A_{2 \max }, A_{2 \min }$ - are the maximum and minimum oscillation amplitudes after installing a test load for measurement from the second side (mm) (Figure 4). 


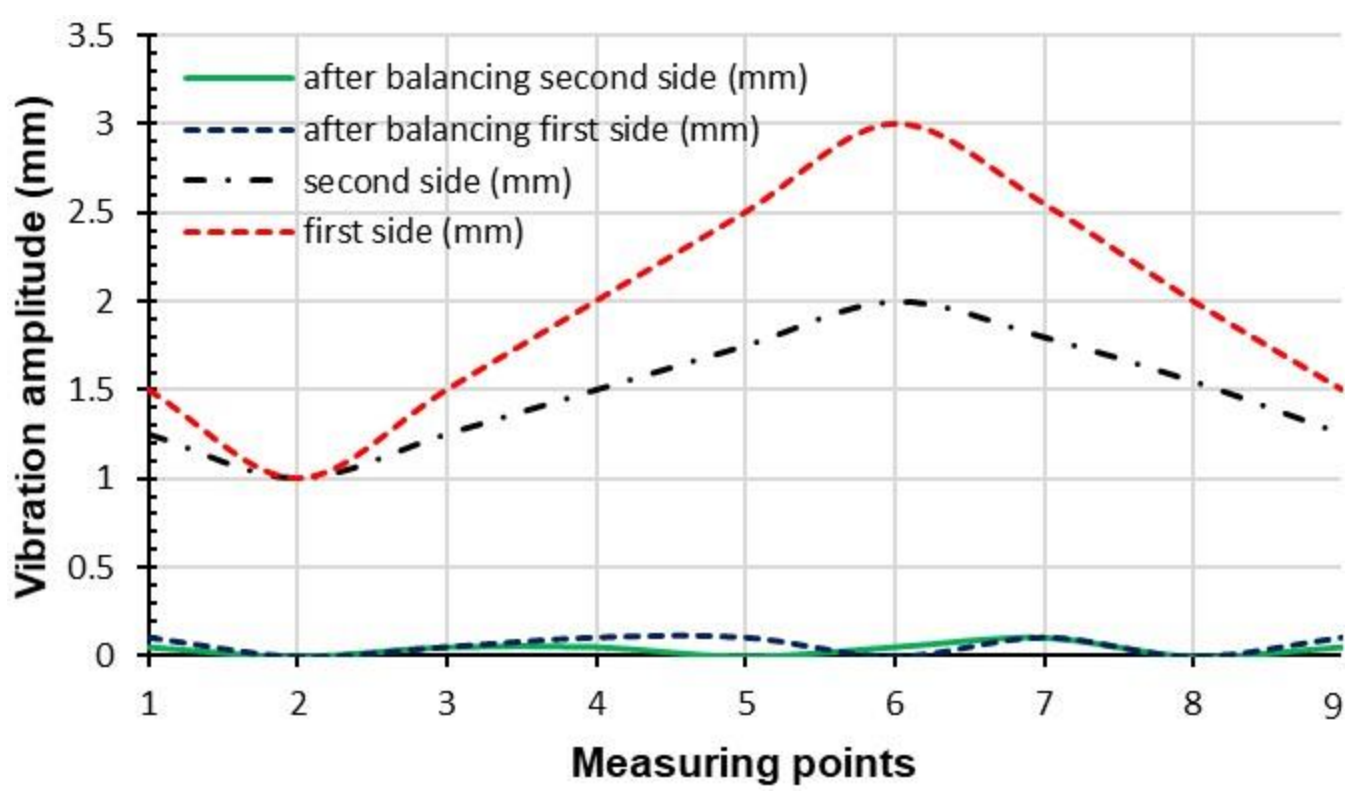

Figure 4 Graph of the parameters of the amplitude of oscillations

In the process of balancing either side, the opposite side loses balance. To compensate for the imbalance in the installation of balancing weights, compensation ones are added to them, which are calculated according to equation (5). After installing the compensation weights, it is necessary to repeat the steps to check the elimination of the unbalanced rotor according to equation (6)

$$
\begin{aligned}
& \Delta G_{1 k}=G_{2 k} \cdot(f /(i+f)) \\
& \Delta G_{2 k}=G_{2 k} \cdot(d \cdot f \cdot((d+i) \cdot(i+f)))
\end{aligned}
$$

where: $d, i, f-$ are the overall distances between the bearings and the rotor( $\mathrm{mm})$

\section{RESULTS}

The experiments on balancing the rotor were carried out on a balancing stand, crushing on a laboratory setup [3]. Having received the data of the unbalanced rotor, performed dynamic balancing of the rotor and received the data, we will draw them on the graph with the data of the unbalanced rotor (Figure 4). After balancing, the curves will be slightly wavy or should be straight (Figure 4).

Parameters of the laboratory setup: internal diameter of the working space $-256 \mathrm{~mm}$, depth of the working space $-60 \mathrm{~mm}$, motor power $-3 \mathrm{~kW}$, engine speed - regulation from $0 \mathrm{~min}^{-1}$ to $3000 \mathrm{~min}^{-1}$; hammers: shape - rectangle, quantity -12 pieces, arrangement - sequential.

To test the crushing of Ni-Al intermetallic, limestone crushed to a final size of 2-3 mm with a total weight of 200 $\mathrm{kg}$ was used as a preliminary material for intermediate experiments [10]. The total weight of the material was divided into two portions of $100 \mathrm{~kg}$ each and passed through a laboratory unit with the registration of the power parameter on the electric motor. At the same time, the first portion, weighing $100 \mathrm{~kg}$, was crushed in a laboratory unit with a balanced rotor, and the second portion, weighing $100 \mathrm{~kg}$, was previously unbalanced. The difference in the indications of the expended power during grinding on a balanced rotor and unbalanced amounted to $8 \%$ in the lower side of the balanced rotor.

When crushing Ni-Al intermetallic, the difference in crushing on a balanced rotor and unbalanced was $15 \%$ [10-12]. 


\section{CONCLUSION}

It is established that one of the negatively influencing factors during the operation of the crusher is structural, namely, the imbalance of the rotor, which manifests itself as a result of uneven wear of the hammers or its design flaws in the manufacturing process. Because of this, energy consumption increases by about $8 \%-15 \%$, crushing time increases and the rate of destruction of critical parts and components of the hammer mill increases. This can be solved by static or dynamic balancing of the crusher rotor. This allows you to reduce the time for maintenance, and accordingly to reduce the technical factor that negatively affects the performance of the crusher and its condition.

Performing balancing actions must be carried out several times during the operation of the crusher, since during natural wear of the working elements of the hammer crusher, vibration will arise and increase. Therefore, it is necessary to prevent its further development in a timely manner.

\section{REFERENCES}

[1] ANDREEV, S.E., PEROV, V.A., ZVEREVICH, V.V. Crushing, Grinding and Screening of Minerals. Moscow: Nedra, 1980.

[2] FISHMAN, M. Impact crushers. Moscow: State Scientific and Technical Publishing House of Mining Literature, 1960.

[3] Ukrainian Patents Database. The stand for research of process of work of a hammer crusher. Patent Number: 121444 [online]. [viewed 2020-06-05]. Available from: http://uapatents.com/5-121444-stend-dlyadoslidzhennya-procesu-roboti-molotkovo-drobarki.html.

[4] ISO 21940-11:2016. Mechanical vibration - Rotor balancing - Part 11: Procedures and tolerances for rotors with rigid behavior [online]. [viewed 2020-06-05]. https://www.iso.org/standard/54074.html.

[5] DIOUF, P., HERBERT, W. Understanding rotor balance for electric motors. In Annual Pulp and Paper Industry Technical Conference. Atlanta: 2014, pp. 7-17.

[6] ROBICHAUD, J., ENG, P. Reference Standards for Vibration Monitoring and Analysis [online]. [viewed 2019-1013]. Available from: http://www.academia.edu/download/33758492/ISO.pdf.

[7] RACIC, Z., HIDALGO, J. Practical balancing of flexible rotors for power generation. Proceedings of the ASME 2007 International Design Engineering Technical: Conferences \& Computers and Information in Engineering Conference IDETC/CIE 2007. Las Vegas: Nevada, USA, 2007.

[8] BRÜEL \& KJ/ER. Static and Dynamic Balancing of Rigid Rotors - A Handbook with author Macdara Maccamhaoil [online]. [viewed 2020-06-05]. Available from: https://www.bksv.com/en/Knowledgecenter/blog/BlogArticles/Vibration-Measurement/static-and-dynamic-balancing-of-rigid-rotors.

[9] SEDUSH, V.Ya. Reliability, Repair and Installation of Metallurgical Machines. Kiev: Polygraphbook, 1976.

[10] BELOKON, Y., ZHEREBTSOV, A., BELOKON, K., FEDCHENOK, A.The investigation of physical-mechanical properties of intermetallic Ni-Al catalyst with nanostructure. In: IEEE International Young Scientists Forum on Applied Physics and Engineering (YSF-2017). 2017. Lviv: IEEE, pp. 299-302.

[11] BELOKON, Y., ZHEREBTSOV, A., BELOKON, K. The investigation of nanostructure formation in intermetallic $\mathrm{Y}^{-}$ TiAl alloys. In: IEEE International Young Scientists Forum on Applied Physics and Engineering (YSF-2017). 2017. Lviv: IEEE, pp. 311-314.

[12] BELOKON, K.V., BELOKON, Y.A., KOZHEMYAKIN, G.B., MATUKHNO, E.V. Environmental assessment of the intermetallic catalysts utilization efficiency for deactivation of the pollutants emitted by electrode production enterprises. Naukovyi Visnyk Natsionalnoho Hirnychoho Universytetu. 2016, vol. 3, pp. 87-94. 